\title{
Coping, schemas, and cardiovascular risks - study protocol
}

\author{
This article was published in the following Dove Press journal: \\ Neuropsychiatric Disease and Treatment \\ 13 October 2017 \\ Number of times this article has been viewed
}

\author{
Milos Slepecky' \\ Antonia Kotianova' \\ Jan Prasko ${ }^{2}$ \\ Ivan Majercak ${ }^{3}$ \\ Erika Gyorgyova ${ }^{4}$ \\ Michal Kotian ${ }^{1,5}$ \\ Marta Zatkova' \\ Ingrid Tonhajzerova ${ }^{6,7}$ \\ Michaela Chupacova ${ }^{1,5}$ \\ Marta Popelkova' \\ 'Department of Psychology Sciences, \\ Faculty of Social Science and Health \\ Care, Constantine the Philosopher \\ University in Nitra, Nitra, Slovak \\ Republic; ${ }^{2}$ Department of Psychiatry, \\ Faculty of Medicine and Dentistry, \\ University Palacky Olomouc, \\ University Hospital Olomouc, \\ Olomouc, Czech Republic; ${ }^{3}$ Ist \\ Department of Internal Medicine, \\ Faculty of Medicine, Pavol Josef \\ Safarik University in Košice, ${ }^{4}$ Internal \\ Medicine and Cardiology Private \\ Practice MUDr Ivan Majercak, \\ Košice, ${ }^{5}$ Psychagogia, Liptovsky \\ Mikulas, ${ }^{6}$ Department of Physiology, \\ ${ }^{7}$ Biomedical Center Martin, Jessenius \\ Faculty of Medicine in Martin, \\ Comenius University in Bratislava, \\ Martin, Slovak Republic
}

Correspondence: Milos Slepecky Department of Psychology Sciences, Faculty of Social Science and Health Care, Constantine the Philosopher University in Nitra, Kraskova I, 94974 Nitra, Slovak Republic

Tel +42I 903500887

Email milos.slepecky@gmail.com

\begin{abstract}
The aim of this article is to describe the protocol of a trial focusing on the psychological, anthropometric, cardiac, and psychophysiological factors contributing to increased risk of cardiovascular diseases (CVDs). As background, the article provides a short overview of research literature linking personal traits, maladaptive schemas, and coping styles with CVDs through reactivity of the autonomic nervous system.
\end{abstract}

Keywords: early maladaptive schemas, coping styles, personality traits, dissociation, heart rate variability

\section{Background}

Cardiovascular diseases (CVDs) cover a range of illnesses related to the circulatory system, including ischemic heart disease (IHD) and cerebrovascular diseases. According to the Organisation for Economic Co-operation and Development (OECD) indicators (2015), CVD remains the leading cause of mortality accounting for nearly one-third (32.3\%) of all deaths in 2013. The Slovak Republic had the highest IHD mortality rate in OECD countries. These results show the need to create a comprehensive approach for prevention and cure. The study INTER-HEART (from 52 countries, with 15,000 patients with acute myocardial infarction and 15,000 matched controls) showed that $90 \%$ of myocardial infarctions in the world could be prevented because they are the result of individuals' lifestyle. ${ }^{1}$ Direct and indirect economic costs and significant deterioration in the quality of life are forcing us to find factors, which can prevent and influence morbidity and mortality. There is also a need for creating programs that can increase the detection of personality traits to cope with emotional burden and stress which can identify the risk factors in human behavior that lead to the onset of CVD.

Distressing situations during childhood or adulthood have been connected with improved CVD risk, although their precise contribution is unknown. ${ }^{2-4}$ In addition, acute psychological stress and feelings of tension and frustration are associated with increased risk of CVD. ${ }^{5-16}$ Similarly, the epidemiological studies showed that psychosocial issues are highly and separately related to the development of CVD. ${ }^{3,17}$ Recently, a meta-analysis of published and unpublished data in over 197,000 participants has shown that job stress is related with a slight, but regular, increased risk of coronary heart disease. ${ }^{16}$

Emotional distress stimulates sympathetic activity producing fluctuations in cardiovascular system - tachycardia, an increase in blood pressure, and relocation of blood flow. ${ }^{18-20}$ The sympathetic hyperactivity accompanied by parasympathetic hypoactivity characterizes the potential pathological mechanism causing heightened 
risk of cardiovascular pathology and also contributes to depression and anxiety disorders..$^{21-24}$

The mean arterial blood pressure remains characteristically controlled within a relatively narrow range at rest and can increase or decrease "appropriately" according to psychological, emotional, or physical stress. ${ }^{20,25}$ Individuals with high baseline sympathetic activity might be at increased risk of hypertension if the "balance" between sympathetic and parasympathetic nervous system is dysregulated. ${ }^{18,26}$ Individuals displaying exaggerated cardiovascular reactivity to stress are at a higher risk of developing heart disease..$^{18,27}$ The study by Lampert et $\mathrm{al}^{28}$ showed that total cumulative stress score and cumulative adverse life events were inversely associated with ultralow-frequency, very low-frequency (VLF), and low-frequency (LF) power and standard deviation of NN intervals.

There is a substantial amount of data connecting sympathetic nervous system hyperresponsivity to the development of hypertension..$^{29,30}$ The studies in patients with borderline hypertension have shown an increased cardiac output, intermediated by increased beta-adrenergic activity and reduced parasympathetic stimulation of the heart. ${ }^{31}$ Elevation of blood pressure was associated with certain personality traits, such as anger and its suppression, and also with unexpected, unfortunate life events. ${ }^{32,33}$ Evidence about the relationship between these factors and hypertension also points to an autonomic nervous system (ANS) dysfunction. ${ }^{31,32}$

The ANS is highly adaptable and permits the organism to continue its balance when experiencing strain or stress. ${ }^{21}$ On the other hand, the lack of flexibility and a rigid ANS can lead to physical and psychological pathologies..$^{24,34-37}$ While various investigations indicate that exaggerated cardiovascular reactivity to stress is linked with cardiovascular complications, ${ }^{29,30}$ a recent study also referred to diminished cardiovascular reactivity as a risk factor for health complications. $^{38}$

Whether cumulative stress, including adversarial life events, is related to decreased heart rate variability (HRV), a noninvasive assessment of autonomic status which reflects poor cardiovascular outcomes, is unknown. HRV could reflect central nervous system activity. ${ }^{39-41}$ Reduced HRV appears to indicate decreased cardiac vagal tone and elevated sympathetic activity in anxious and depressive patients and would reflect a deficit in the flexibility of emotional and physiological mechanisms. ${ }^{35}$ Total cumulative stress and adverse life events are connected with reduced cardiac autonomic function as measured by HRV. ${ }^{28}$ The decreased HRV is associated with other CVD risk factors. ${ }^{42}$ Low HRV is linked with higher risk of the first cardiovascular incident in persons without known CVD. ${ }^{43}$

Psychological aspects and stressful life events play a role at the beginning of the metabolic syndrome associated with several diseases (eg obesity), which is the risk factor for CVD. ${ }^{44}$ It is supposed that higher body weight is associated with clinical CVDs even at moderate degrees as an independent risk factor. ${ }^{45,46}$ The pathophysiological mechanisms of cardiovascular dysfunction in obesity have not been fully understood yet, but autonomic cardiovascular dysregulation has been anticipated as a link connecting higher body weight and the increased prevalence of heart disease ${ }^{47}$ Earlier studies using HRV methods to measure cardiovascular autonomic function have shown that obese persons have a significant decrease in parasympathetic activity and a significant elevation in sympathetic modulation of cardiac function, demonstrating a shift in the sympathovagal equilibrium towards sympathetic predominance. ${ }^{48,49}$

\section{Preliminary study}

We have finalized a small pre-pilot study to assess the feasibility of design and the appropriateness of the study protocol..$^{50} \mathrm{We}$ compared the psychological, psychophysiological, and anthropometric factors connected with life events, depressivity, and quality of life of the persons with the risk for the development of CVD and healthy controls. The patients with CVDs showed higher scores of life events and depression and lower quality of life than healthy controls. In healthy controls, a higher amount of life events significantly negatively correlated with high-frequency total power, a possible marker of parasympathetic tone, and the level of depression correlated with overweight. In patients with cardiovascular problems, the score of life events was not linked with the cardiovascular or anthropometric factors. The main limitation of this study was the number of probands, but the results suggest some potential mechanisms by which stress may exert adversarial effects on cardiovascular morbidity in healthy persons.

\section{Study aims}

The study aims to determine whether psychological variables are related to risk aspects for CVD, and if so, what is the relationship and how this relation is mediated.

\section{Expected model}

The possible complex relations are shown in Figure 1. 


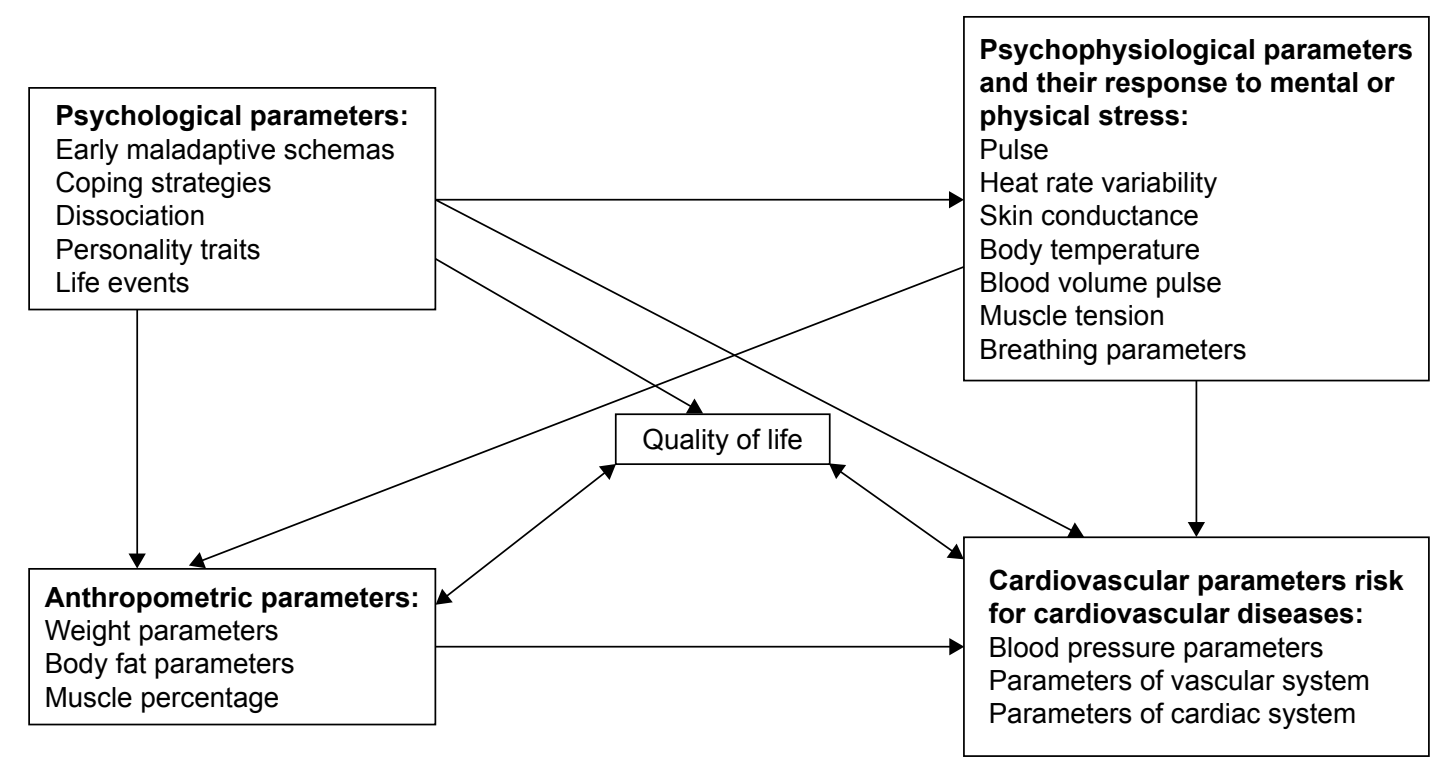

Figure I Complex model of influences between important factors leading to cardiovascular parameters risk for cardiovascular diseases.

\section{Theoretical hypotheses}

(1) There is a relationship between psychological parameters and risk factors for CVDs.

(2) Psychological parameters are related to psychophysiological parameters and their responses to mental and physical stress.

(3) Psychological parameters are related to anthropometric parameters.

(4) Psychological parameters are associated with cardiovascular parameters risk for CVDs.

(5) Psychophysiological parameters are related to cardiovascular parameters risk for CVDs.

(6) Anthropometric parameters are linked to cardiovascular parameters risk for CVDs.

(7) There exists a model that explains the pathway among psychological, psychophysiological, and anthropometric parameters risk factors and cardiovascular parameters risk for CVDs.

\section{Methods}

A cross-sectional study will be conducted to collect data for the evaluation of the relationship between psychological characteristics and the early indicators of CVD.

\section{Participants and study setting}

The study recruited 200 healthy persons aged above 21 years taking no medication that can influence the ANS.

The probands will be evaluated under standard conditions from 8.00 to 13.00 hours in the outpatient cardiology clinic.
During first 15 minutes, participants will be informed about the protocol, and informed consent will be obtained. This time will also allow the probands to adapt to the environment and to calm down. Following this, the cardiological, psychological, psychophysiological, and anthropometric measurements will be taken.

The study will follow the rules outlined in the study by Farsky: ${ }^{51}$ the room temperature should be $22\left( \pm 1^{\circ} \mathrm{C}\right)$, after 10 -minute rest. The probands can have only simple breakfast, and smoking and coffee drinking are not allowed 6 hours before the assessment. Alcohol drinking is forbidden 10 hours before measurements. Probands should have enough sleep the night before measurement. During measurement, sleeping and speaking are not allowed. The room should be free from outside sounds.

\section{Measurements \\ Questionnaires}

The following questionnaires will be used in the study.

Young Schema Questionnaire (YSQ-S3)

YSQ-SF3 was developed by Young. $.52,53$ Ninety items are rated on a 6-point Likert-type scale. As each subscale consists of 5 items, the score obtained on the subscales varies between 5 and 30. The questionnaire has 18 subscales grouped into 5 schema domains as follows: Rejection, Impaired autonomy and performance, Impaired limits, Other-directedness, Over-vigilance and inhibition. Psychometric assessment using this questionnaire has been done in several countries. In studies from Turkey, Cronbach's $\alpha$ internal consistency coefficients 
of schema domains determined by higher-order factor analysis ranged between 0.53 and 0.81 . Internal consistency of 17 of the 18 scales was sufficient (Cronbach's $\alpha>0.70$ ). Internal consistency of the scale "Entitlement/grandiosity" was $0.67 . .^{54-56}$ The purpose of the ongoing study is to perform psychometric assessment in Slovakia using this questionnaire.

\section{Young-Rygh Avoidance Questionnaire (YRAQ)}

YRAQ is a 41-item questionnaire which assesses schema Avoidance. ${ }^{57}$ Individuals rate responses on a 6-point scale. The high total score indicates a general pattern of schema avoidance. The inventory is not schema-specific: an avoidant coping style is often a pervasive trait that can be utilized to avoid any scheme. The reliability coefficient obtained through splitting was 0.79 in an Iranian study. ${ }^{58}$

\section{Young Compensation Inventory $(\mathrm{YCl})$}

YCI is a 48-item questionnaire that assesses schema overcompensation. The inventory has adequate test-retest reliability $^{59}$ and high internal consistency. ${ }^{60,61}$

Temperament and Character Inventory - Revised (TCl-R) TCIR consists of 240 items. $^{62}$ The questionnaire evaluates 4 temperamental and 3 character personality traits. Features of temperament include Novelty seeking, Harm avoidance, Reward dependence, and Persistence. Characteristic features include Self-directedness, Cooperativeness, and Self-transcendence. ${ }^{63}$ Czech percentile standards were created by Preiss and Klose. ${ }^{64}$

\section{Dissociative Experiences Scale (DES)}

DES describes 28 dissociative experiences. Patients mark a spot on a $10-\mathrm{cm}$ line according to the frequency of symptoms they experience. ${ }^{65}$ Current modifications of the dissociation model have arrived at the difference between a dimensional, nonpathological type and a discontinuous, pathological class of dissociation, which can be recognized by a subgroup of 8 items of the DES, the DES-Taxon (DES-T). ${ }^{66}$ This subscale consists of 8 out of the 28 DES items (items 3, 5, 7, 8, 12, 13, 22, and 27). ${ }^{67}$ The Czech version of the scale is similar to the original version regarding its validity, test-retest reliability, and the factor structure. ${ }^{68}$

\section{Type-D Scale (DS- I4)}

D-type personality is defined as an increased tendency to experience negative emotions (negative affectivity, NA) in connection to non-expressed emotions (social inhibition, SI). ${ }^{69}$ NA is connected with the tendency to experience negative emotions like anxiety, anger, hostility, irritability, and dysphoria.
SI refers to the difficulties in emotion expression and discomfort in the social situations. Type D is considered to be a relatively stable personal characteristic. ${ }^{69}$ In 1998, Johan Denollet developed DS-14 questionnaire based on the items from Minnesota Multiphasic Personality Inventory and the items created specifically to determine D-type personality. Scale DS-14 consists of 14 items, 8 of them are related to NA and 6 to SI. On a sample of 400 people, the results confirmed that the scale was internally consistent (Cronbach's $\alpha 0.89$ [NA]; 0.82 [SI]) and stable over time (test-retest after 3 months: 0.78 [NA]; 0.87 [SI]). ${ }^{70}$ Slovak adaptation of the scale was made by Durka. ${ }^{71,72}$ Psychometric properties were good, with Cronbach's $\alpha=0.89$ and 0.86 for NA and SI, respectively. ${ }^{73}$

\section{Demographic questionnaire (DD)}

DD contains basic information such as sex, age, marital and partnership status, employment status, education, and pension status. The questionnaire was developed for requirements of this study.

\section{Psychophysiological measure}

Heart rate variability as a psychophysiological measure will be evaluated. The differences in the ANS activity, indexed by HRV, have been measured by the analytic system ProComp Infiniti which uses the power spectral analysis of the beat-tobeat time series and quantifies the HRV. HRV was assessed in 3 positions (first -5 minutes supine; second -5 minutes standing; third - 5 minutes supine), recording beat-to-beat interval during paced breathing by ECG sensor with UniGel electrodes snapped on and placed on the chest. Power spectra were computed by a fast Fourier transform system for 3 spectrum ranges: (1) VLF (0.0033-0.04 Hz), (2) LF (0.04-0.15 Hz), and (3) high-frequency (HF: $0.15-0.40 \mathrm{~Hz}$ ) powers. ${ }^{74-77}$ To examine heart rate, we used the microcomputer system ProComp Infiniti. The system allows the transmission of the ECG signal radio to the receiver connected to the PC. Information was processed by a software program Biograph Infinity (version 6) from Thought Technology.

\section{Anthropometric characteristics}

Basic anthropometric parameters will be measured: weight, height, hip size, waistline, waist/hip ratio, waist/height ratio, and whole-body dual-frequency segmental bio-impedancemuscle mass, fat stock, \% body fat, segmental distribution of muscle and fat mass, and fat-free mass.

\section{Cardiovascular parameters}

Basic cardiovascular parameters - blood pressure ( $\mathrm{mmHg}$ ) and heart rate (bpm) - will be evaluated at rest and in response 
to the orthostatic test. Using arteriography (Medexpert arteriography), aortic pulse wave velocity (PWVA), central aortic pressure, and augmentation index (AIX) will be measured for assessment of the risk of atherosclerosis. In addition, the stiffness/elasticity of the arteries (PWVA, AIX) will be measured and ECG (detection of QTc interval in $\mathrm{ms}$ ) will be examined for estimating cardiovascular risk.

\section{Primary outcome measures}

Primary outcome measures indicating the cardiovascular parameters risk for CVDs are the stiffness/elasticity of the arteries (PWVA, AIX), blood pressure, and QTc interval.

\section{Data monitoring}

The data will be collected continuously and immediately entered into an Excel table by the study researchers. All data will be double-checked monthly for accuracy, referring to the paper-based forms. The data will be protected following the completion of the examination of the last participant at the final time point.

All databases will be organized according to the unique participant ID number (assigned at the beginning of the study), ensuring that the databases are merged correctly.

\section{Statistical analysis}

Statistics will be calculated using statistical software SPSS 24.0 for Windows and GraphPad Prism (version 5.0; http:// www.graphpad.com/prism/prism.htm). The following statistical measures will be calculated: descriptive statistics for the demographic data, mean scores, and character of data distribution. Demographic, anthropometric, physiological, and psychological data will be analyzed using column statistics. The Shapiro-Wilk $W$-test will be used to determine normal distribution of the demographic and clinical variables. Differences between scores or measures will be computed by $t$-tests or Mann-Whitney tests in alternative subgroups of data, one-way ANOVA, or Kruskal-Wallis test in more than 2 data categories, according to the type of data distribution. The Fisher's exact tests and chi-square tests will be used for the categorical variables. Relationships between treatment outcome and other factors will be analyzed by Pearson or Spearman and partial correlations. For reduction of the multicollinearity, the regression analysis will be made. Differences will be considered significant if $p$-values are less than 0.05 .

\section{Ethical issues}

The investigation will be performed in agreement with the latest version of the Declaration of Helsinki and the Guideline for Good Clinical Practice. ${ }^{78}$ The local ethical committee of the Faculty of Social Science and Health Care, Constantine the Philosopher University in Nitra approved the study. Written informed consent will be obtained from participants after the procedures are fully explained.

\section{Trial status}

Participants began to enter the trial in January 2017. Recruitment will continue until December 2017.

\section{Timetable}

(1) Preparing phase: July 2016-November 2016

(2) Recruitment of the patients, data collection and ongoing analyses of the results, and publishing of partial results: November 2016-July 2018

(3) Analysis of the results and publishing: August 2018December 2020.

\section{Discussion}

The results of the study can help in better understanding of the interface between psychological parameters, psychophysiological parameters, anthropometric characteristics, and cardiovascular parameters risk for CVDs in individuals who were not treated for CVDs yet. These results may help to find new diagnostic, preventive, and treatment approaches.

There are various limitations linked to the technical and human possibilities of the probands, researchers, planned methods, and devices. The first is the high number of questionnaires which the probands have to fulfill. The problem of concentration will rise with time. The plan is exchanged between working with "papers" and cardiological and anthropometric measurements. We offer the probands the possibility to look for the outcomes and write summaries of the most relevant results and also give them expert advice in cases of recognized risk factors.

\section{Conclusion}

This study could find the correlation between psychological parameters, psychophysiological parameters, anthropometric parameters, and cardiovascular parameters risk for CVDs in asymptomatic individuals and create a possible model of interactions between parameters. Especially, this study will give an insight into the influences of early maladaptive schemas, coping styles, and personality traits on the risk for CVD. These findings could influence the development of new psychological and lifestyle-changing strategies for the prevention of CVD.

\section{Acknowledgment}

This article was supported by the research grant VEGA no APVV-15-0502. 


\section{Disclosure}

The authors report no conflicts of interest in this work.

\section{References}

1. Scheen AJ, Kulbertus H. Interheart: nine risk factors predict nine out of ten myocardial infarctions. Rev Med Liege. 2004;59(11):676-679.

2. Everson-Rose SA, Lewis TT. Psychosocial factors and cardiovascular diseases. Annu Rev Public Health. 2005;26:469-500.

3. Rozanski A, Blumenthal JA, Kaplan J. Impact of psychological factors on the pathogenesis of cardiovascular disease and implications for therapy. Circulation. 1999;99(16):2192-2217.

4. Leor J, Poole WK, Kloner RA. Sudden cardiac death triggered by an earthquake. N Engl J Med. 1996;334(7):413-419.

5. Mostofsky E, Maclure M, Sherwood JB, Tofler GH, Muller JE, Mittleman MA. Risk of acute myocardial infarction after the death of a significant person in one's life: the Determinants of Myocardial Infarction Onset Study. Circulation. 2012;125(3):491-496.

6. Gullette EC, Blumenthal JA, Babyak M, et al. Effects of mental stress on myocardial ischemia during daily life. JAMA. 1997;277(19): $1521-1526$.

7. Iso $\mathrm{H}$, Date $\mathrm{C}$, Yamamoto A, et al. Perceived mental stress and mortality from cardiovascular disease among Japanese men and women: the Japan collaborative cohort study for evaluation of cancer risk sponsored by Monbusho (JACC Study). Circulation. 2002;106(10):1229-1236.

8. Edmondson D, Cohen BE. Posttraumatic stress disorder and cardiovascular disease. Prog Cardiovasc Dis. 2013;55(6):548-556.

9. Aboa-Eboulé C, Brisson C, Maunsell E, et al. Job strain and risk of acute recurrent coronary heart disease events. JAMA. 2007;298(14): $1652-1660$.

10. Bugajska J, Widerszal-Bazyl M, Radkiewicz P, et al. Perceived workrelated stress and early atherosclerotic changes in healthy employees. Int Arch Occup Environ Health. 2008;81(8):1037-1043.

11. Hintsanen M, Kivimäki M, Elovainio M, et al. Job strain and early atherosclerosis: the cardiovascular risk in Young Finns Study. Psychosom Med. 2005;67(5):740-747.

12. Lynch J, Kaplan GA, Salnone R, Salonen JT. Socioeconomic status and progression of carotid atherosclerosis. Prospective evidence from the Kuopio Ischemic Heart Diseases Risk Factor Study. Arterioscler Thromb Vasc Biol. 1997;17(3):513-519.

13. Michikawa T, Nishiwaki Y, Nomiyama T, Uemura T, et al. Job strain and arteriosclerosis in three different types of arteries among male Japanese factory workers. Scand J Work Environ Health. 2008;34(1):48-54.

14. Nomura K, Nakao M, Karita K, Nishikitani M, Yano E. Association between work-related psychological stress and arterial stiffness measured by brachial-ankle pulse-wave velocity in young Japanese males from an information service company. Scand J Work Environ Health. 2005;31(5):352-359

15. Nordstrom CK, Dwyer KM, Merz CN, Shircore A, Dwyer JH. Workrelated stress and early atherosclerosis. Epidemiology. 2001;12(2): $180-185$.

16. Kivimäki M, Head J, Ferrie JE, et al. Work stress, weight gain and weight loss: evidence for bidirectional effects of job strain on body mass index in the Whitehall II study. Int J Obes (Lond). 2006;30(6):982-987.

17. Hemingway H, Malik M, Marmot M. Social and psychosocial influences on sudden cardiac death, ventricular arrhythmia, and cardiac autonomic function. Eur Heart J. 2001;22(10):82-101.

18. Krantz DS, Manuck SB. Measures of acute physiologic reactivity to behavioral stimuli: assessment and critique. Psychol Bull. 1984;96:435-464.

19. Fontes MA, Xavier CH, de Menezes RC, DiMicco JA. The dorsomedial hypothalamus and the central pathways involved in the cardiovascular response to emotional stress. Neuroscience. 2011;184:64-74.

20. Zanstra YJ, Johnston DW. Cardiovascular reactivity in real life settings: measurement, mechanisms, and meaning. Biol Psychol. 2011;86(2): 98-105.
21. Jones-Webb R, Jacobs DR Jr, Flack JM, Liu K. Relationship between depressive symptoms, anxiety, alcohol consumption, and blood pressure: results from the CARDIA study. Alcohol Clin Exp Res. 1996;20(3): 420-427.

22. Prasko J, Latalova K, Diveky T, et al. Panic disorder, autonomic nervous system, and dissociation - changes during therapy. Neuro Endocrinol Lett. 2011;32(5):641-651.

23. Latalova K, Prasko J, Diveky T, et al. Autonomic nervous system in euthymic patients with bipolar affective disorder. Neuro Endocrinol Lett. 2010;31(6):829-836.

24. Diveky T, Prasko J, Kamaradova D, et al. Comparison of heart rate variability in patients with panic disorder during cognitive behavioral therapy program. Psychiatr Danub. 2013;25(1):62-67.

25. Joyner MJ, Charkoudian N, Wallin BG. A sympathetic view of the sympathetic nervous system and human blood pressure regulation. Exp Physiol. 2008;93(6):715-724.

26. Johnston DW, Tuomisto MT, Patching GR. The relationship between cardiac reactivity in the laboratory and in real life. Health Psychol. 2008; 27(1):34-42.

27. Treiber FA, Kamarck T, Schneiderman N, Sheffield D, Kapuku G, Taylor T. Cardiovascular reactivity and development of preclinical and clinical disease states. Psychosom Med. 2003;65(1):46-62.

28. Lampert R, Tuit K, Hong KI, Donovan T, Lee F, Sinha R. Cumulative stress and autonomic dysregulation in a community sample. Stress. 2016; 19(3):269-279.

29. Lovallo WR, Gerin W. Psychophysiological reactivity: mechanisms and pathways to cardiovascular disease. Psychosom Med. 2003;65(1): $36-45$.

30. Grassi G, Bombelli M, Brambilla G, Trevano FQ, Dell'oro R, Mancia G. Total cardiovascular risk, blood pressure variability and adrenergic overdrive in hypertension: evidence, mechanisms, and clinical implications. Curr Hypertens Rep. 2012;14(4):333-338.

31. Light KC. Psychosocial precursors of hypertension: experimental evidence. Circulation. 1987;76(1 Pt 2):67-76.

32. James SA. Psychosocial precursors of hypertension: a review of the epidemiologic evidence. Circulation. 1987;76(1 Pt 2):I60-I66.

33. Buck CW, Donner AP. Blood pressure control in hypertensives - a model for the study of life events. J Chronic Dis. 1984;37(4):247-253.

34. Sloan RP, Shapiro PA, Baggiela E, Myers MM, Gorman JM. Cardiac autonomic control buffers blood pressure variability response to challenge: a psychophysiologic model of coronary artery disease. Psychosom Med. 1999;61(1):58-68.

35. Visnovcova Z, Calkovska A, Tonhajzerova I. Heart rate variability and electrodermal activity as noninvasive indices of sympathovagal balance in response to stress. Acta Med Martiniana. 2013;13(1):5-13.

36. Visnovcova Z, Mestanik M, Gala M, Mestanikova A, Tonhajzerova I. The complexity of electrodermal activity is altered in mental cognitive stressors. Comput Biol Med. 2016;79:123-129.

37. Jelenova D, Ociskova M, Prasko J, et al. Heart rate variability in children with inflammatory bowel diseases. Neuro Endocrinol Lett. 2015;36(1):72-79.

38. Lovallo WR. Do low levels of stress reactivity signal poor states of health? Biol Psychol. 2011;86(2):121-128.

39. Benarroch EE. The central autonomic network: functional organization, dysfunction, and perspective. Mayo Clin Proc. 1993;68(10): 988-1001.

40. Thayer JF, Lane RD. A model of neurovisceral integration in emotional regulation and dysregulation. J Affect Disord. 2000;61(3):201-216.

41. Thayer JF, Lane RD. Claude Bernard and the heart-brain connection: further elaboration of a model of neurovisceral integration. Neurosci Biobehav Rev. 2009;33(2):81-88.

42. Colhoun HM, Francis DP, Rubens MB, Underwood SR, Fuller JH. The association of heart-rate variability with cardiovascular risk factors and coronary artery calcification: a study in type 1 diabetic patients and the general population. Diabetes Care. 2001;24(6):1108-1114. 
43. Hillebrand S, Gast KB, de Mutsert R, et al. Heart rate variability and first cardiovascular event in populations without known cardiovascular disease: meta-analysis and dose-response meta-regression. Europace. 2013;15(5):742-749.

44. Fabre B, Grosman H, Mazza O, et al. Relationship between cortisol, life events and metabolic syndrome in men. Stress. 2013;16(1):16-23.

45. Prospective Studies Collaboration. Body-mass index and cause-specific mortality in 900000 adults: collaborative analyses of 57 prospective studies. Lancet. 2009;373(9669):1083-1096.

46. Chen Z, Yang G, Zhou M, et al. Body mass index and mortality from ischaemic heart disease in a lean population: a 10-year prospective study of 220,000 adult men. Int J Epidemiol. 2006;35(1):141-150.

47. Kim JA, Park YG, Cho KH, et al. Heart rate variability and obesity indices: emphasis on the response to noise and standing. J Am Board Fam Pract. 2005;18(2):97-103.

48. Rabbia F, Silke B, Conterno A, et al. Assessment of cardiac autonomic modulation during adolescent obesity. Obes Res. 2003;11(4):541-548.

49. Chethan HA, Niranjan M, Basavaraju K. Comparative study of heart rate variability in normal and obese young adult males. Int J Biol Med Res. 2012;3:1621-1623.

50. Slepecky M, Kotianova A, Prasko J, et al. Which psychological, psychophysiological, and anthropometric factors are connected with life events, depression, and quality of life in patients with cardiovascular disease. Neuropsychiatr Dis Treat. 2017;13:2093-2104.

51. Farsky S. Vyšetrovanie arteriálnej tuhosti [Investigation of arterial stiffness]. Via Pract. 2009;6(4):153-156. Slovak [with English abstract].

52. Young JE. Cognitive therapy for personality disorders: a schema-focused approach. 2nd ed. Sarasota, FL: Proffesional Resource Exchange; 1994.

53. Young JE, Weishaar ME, Klosko JS: Schema Therapy: A Practitioner's Guide. New York, Ny: Guilford; 2003.

54. Cecero JJ, Nelson JD, Gillie JM. Tools and tenets of schema therapy: toward the construct validity of the early maladaptive schema questionnaire - research version (EMSQ-R). Clin Psychol Psychother. 2004; $11: 344-357$

55. Soygüt G, Karaosmanoğlu A, Çakir Z. Assessment of early maladaptive schemas: a psychometric study of the Turkish Young schema questionnaire-short form-3. Turk Psikiyatri Derg. 2009;20(1):75-84.

56. Kriston L, Schäfer J, Jacob GA, Härter M, Hölzel LP. Reliability and validity of the German version of the Young Schema QuestionnaireShort Form 3 (YSQ-S3). Eur J Psychol Assess. 2013;29(3):197-204.

57. Young JE, Rygh J. Young-Rygh Avoidance Inventory. New York: Cognitive Therapy Center of New York; 1994.

58. Bayrami M, Bakhshipor A, Esmaeili A. The relationship between coping styles and early maladaptive schemas in disconnection-rejection and over vigilance - inhibition in Young's schema model. J Life Sci Biomed. 2012;2(4):178-181.

59. Rijkeboer MM, van den Bergh H, van den Bout J. Stability and discriminative power of the Young Schema-Questionnaire in a Dutch clinical versus non-clinical population. J Behav Ther Exp Psychiatry. 2005;36(2):129-144.

60. Lee CW, Taylor G, Dunn J. Factor structure of schema questionnaire in a large clinical sample. Cognit Ther Res. 1999;23:441-451.

61. Baranoff J, Oei TP, Cho SH, Kwon SM. Factor structure and internal consistency of the Young Schema Questionnaire (Short Form) in Korean and Australian samples. J Affect Disord. 2006;93(1-3):133-140.
62. Farmer RF, Goldberg LR. A psychometric evaluation of the revised Temperament and Character Inventory (TCI-R) and the TCI-140. Psychol Assess. 2008;20(3):281-291.

63. Gillespie NA, Cloninger CR, Heath AC, Martin NG. The genetic and environmental relationship between Cloninger's dimensions of temperament and character. Pers Individ Dif. 2003;35(8):1931-1946.

64. Preiss M, Klose J. Diagnostika poruch osobnosti pomocí teorie C.R. Cloningera [Diagnostic of personality disorders using theory of C.R. Cloninger]. Psychiatrie. 2001;5:226-231. Czech [with English abstract].

65. Bernstein EM, Putnam FW. Development, reliability, and validity of a dissociation scale. J Nerv Ment Dis. 1986;174(12):727-735.

66. Spitzer C, Freyberger H, Brähler E, Beutel ME, Stieglitz R. Psychometric evaluation of the Dissociative Experiences Scale-Taxon (DES-T). Psychother Psychosom Med Psychol. 2015;65(3-4):134-139.

67. Waller NG, Ross CA. The prevalence and biometric structure of pathological dissociation in the general population: taxometric and behavior genetic findings. J Abnorm Psychol. 1997;106(4):499-510.

68. Ptacek R, Bob P, Paclt I. Škála disociativních zkušeností - česká verze [Dissociative Experiences Scale - Czech version]. Česk Psychol. 2006;50(3):262-272. Czech [with English abstract].

69. Denollet J. Standard assessment of negative affectivity, social inhibition, and Type D personality. Psychosom Med. 2005;67(1):89-97.

70. Denollet J. Personality and coronary heart disease: the type-D scale-16 (DS16). Ann Behav Med. 1998;20(3):209-215.

71. Durka R. Osobnost' typu D [Personality Type D]. In: Selko D, editor. Psychologie zdravia. Bratislava: Mauro; 2006:86-93. Slovak.

72. Durka R. Osobnost' typu D u dospelých [Personality Type D in adults]. In: Heller D, Michalek P, editors. Cesty psychologie a psychologie cest. Praha: Českomoravská psychologická společnost; 2011:532-543. Slovak.

73. Durka R, Ruch W. Osobnost' typu D v populácii Slovenských univerzitných študentov: psychometrické kvality dotazníka DS14 [Personality of Type D population of Slovak University students: psychometric quality of the questionnaire DS14]. Ceskoslovenská psychológie. 2014;58(1):62-71. Slovak [with English abstract].

74. Lipsitz LA, Mietus J, Moody GB, Goldberger AL. Spectral characteristics of heart rate variability before and during a postural tilt: relations to aging and risk of syncope. Circulation. 1990;81:1803-1810.

75. Weise F, Heydenreich F, Runge U. Contributions of sympathetic and vagal mechanisms to the genesis of heart rate fluctuations during orthostatic load: a spectral analysis. J Auton Nerv Syst. 1987;21(2-3): $127-134$.

76. Ponikowski P, Chua TP, Piepoli M, et al. Chemoreceptor dependence of very low frequency rhythms in advanced chronic heart failure. Am J Physiol. 1997;272(1 Pt 2):H438-H447.

77. Javorka K. Heart Rate Variability. Mechanisms, Classification, Clinical Utilization. Martin: Osveta; 2008.

78. European Medicines Agency. Reflection paper on ethical and GCP aspects of clinical trials of medicinal products for human use conducted in third countries and submitted in marketing authorisation applications to the EMA [Draft]. Available from: http://www.ema.europa.eu/pdfs/ human/ich/013595en.pdf. Accessed October 5, 2017.
Neuropsychiatric Disease and Treatment

\section{Publish your work in this journal}

Neuropsychiatric Disease and Treatment is an international, peerreviewed journal of clinical therapeutics and pharmacology focusing on concise rapid reporting of clinical or pre-clinical studies on a range of neuropsychiatric and neurological disorders. This journal is indexed on PubMed Central, the 'PsycINFO' database and CAS,

\section{Dovepress}

and is the official journal of The International Neuropsychiatric Association (INA). The manuscript management system is completely online and includes a very quick and fair peer-review system, which is all easy to use. Visit http://www.dovepress.com/testimonials.php to read real quotes from published authors. 